\title{
The Emergence of Omicron: Challenging Times Are Here Again!
}

\author{
Tanu Singhal ${ }^{1}$
}

Received: 31 December 2021 / Accepted: 3 January 2022 / Published online: 13 January 2022

(c) Dr. K C Chaudhuri Foundation 2022

\begin{abstract}
COVID-19 has been reported to have caused more than 286 million cases and 5.4 million deaths till date. COVID variants have appeared at regular intervals-alpha, beta, gamma, delta and now omicron. 'Omicron' is driving the current surge of cases in most countries including India and is poised to replace 'delta' the world over. This variant with more than 50 mutations is phylogenetically very different from other variants. The omicron variant spreads rapidly with an average doubling time of two days. The disease so far has been mild as compared with delta. Though previous infection and vaccination offer little or no protection against infection with omicron, they do seem to partially protect against hospitalization and severe disease. Booster vaccinations have not made any notable impact on the spread of omicron and have further worsened global vaccine equity. The indirect consequences of omicron from lockdowns, restrictions, travel bans, economic losses, health care worker infections and overwhelming of health care facilities are likely to be enormous. The direct effects of omicron on children are expected to be mild like with the previous variants. However, the indirect effects on child mental, physical, and social health may be considerable owing to school closures, missed vaccinations, neglect of other diseases, etc. It is, therefore, imperative that governments take rational decisions to navigate the world through this latest crisis.
\end{abstract}

Keywords COVID-19 · Children · Omicron variant · Variant of concern (VOC) · Third wave

\section{Introduction}

The COVID-19 pandemic, the biggest public health crises in a century has caused an estimated 286 million cases and 5.4 million deaths till date [1]. As the year 2021 was drawing to a close, with increasing natural and vaccine-induced immunity, the general view was that the worst is over and an end to the pandemic is in sight. The world was reopening again. But with emergence of the omicron variant, panic and fear has set in with a concern that all the gains achieved so far may be negated [2]. This article summarizes the current knowledge about omicron and then discusses briefly the current status of the COVID-19 pandemic in children. Since this is a rapidly evolving situation, readers are requested to update themselves regularly. As most of the information is from lay media and nonpeer reviewed preprints, careful interpretation is required.

Tanu Singhal

tanusinghal@yahoo.com

1 Department of Pediatrics and Infectious Disease, Kokilaben Dhirubhai Ambani Hospital and Medical Research Institute, Mumbai, Maharashtra 400053, India

\section{Omicron-An Overview}

\section{Origin and Spread of Omicron}

In November 2021, researchers in Botswana and South Africa on whole-genome sequencing (WGS) identified a SARS-CoV-2 variant. It stood out, as it had more than 50 mutations in its genome; of which, 32 were in the receptor binding domain (RBD) of the spike protein [3]. At the same time, there was a rapid increase in COVID-19 cases in South Africa, particularly the Gauteng province of Johannesburg; many of which were associated with S-gene target failure (SGTF) on the Taqpath RT-PCR test [4]. This variant was reported by South Africa to the WHO on 24th November, 2021. Almost simultaneously, isolates with similar genomic sequence were identified in Belgium, Israel, and Hong Kong [4]. Taking cognizance of the significant numbers of mutations and rapid rise of infections in South Africa, the WHO labelled this variant just $2 \mathrm{~d}$ later, on 26th November, as a variant of concern (VOC) and christened it omicron [5]. This led to many nations including India imposing a travel ban on African nations despite the fact that omicron cases were simultaneously identified in many other European countries 
suggesting that the variant was widespread in many countries even before it was identified [6].

Subsequently, the cases in South Africa, which had peaked to 40,000 per day have started coming down rapidly in less than a month to 7,000 per day [7]. But omicron has spread rapidly through the world and is fueling waves in other countries. S-gene target failure (SGTF) as a proxy for omicron, is allowing rapid identification of probable omicron cases pending genomic sequencing [8]. UK is severely affected with more than 100,000 cases per day; in London more than $90 \%$ of the patients are infected with omicron [9]. Similar waves are occurring in France, Germany, the Netherlands, and other European countries fueling lockdowns, restrictions, and a push for booster vaccinations [10]. The variant has rapidly spread to the US adding to the ongoing delta wave [10]. On 29th December 2021 there were more than 400,000 daily COVID-19 cases in the US with omicron causing 58\% of all COVID-19 cases [11]. Omicron is now reported in more than 100 countries and caused the global case count to exceed 1 million daily cases on 28th December 2021 [1].

Omicron has been detected in India as well, initially in patients who had returned from international travel and now also in those with no travel history. There is a rapid rise in infections in Mumbai and Delhi in the past few days [12]. The third wave of COVID-19 in India fueled by omicron is here.

\section{Virology}

Omicron shares mutations with the alpha, beta, gamma, and delta variants and others of unknown significance [3]. The characteristics of some of these mutations are still being unraveled. Within a couple of months of its emergence, it has already split into two lineages [13]. The behavior of omicron in cell culture is interesting [14]. Scientists have reported that omicron is difficult to grow in VERO cells (genetically engineered African green monkey kidney cells) like other seasonal coronaviruses and unlike SARS-CoV-2. Besides, it is less likely to cause visible damage (plaques) in these cells. Also, the ability to cause syncytia formation which is are often seen in the lungs of patients dying of COVID-19 is less with omicron. Researchers from Hong Kong have reported a 10 times faster growth in bronchi as compared to delta (accounting for rapid spread) but a tenfold slower growth in the lungs [14]. While it is still early days, these observations may account for milder disease seen with omicron.

\section{Transmissibility}

Omicron is a very highly transmissible variant with studies from South Africa and UK reporting doubling times of $3.38 \mathrm{~d}(95 \%$ CI $3.18-3.61 \mathrm{~d})$ and 2-2.5 d, respectively [15, $16]$ with the basic reproduction number $\left(\mathrm{R}_{0}\right)$ above 3 . This property is accounting for its rapid spread and displacement of the prevailing delta variant.

\section{Clinical Features and Complications}

Preliminary information about omicron suggests that the median incubation period may be shorter at around 2-3 d as compared to the $5 \mathrm{~d}$ with the original virus [17]. The usual symptoms reported with omicron are predominantly upper respiratory and include runny nose, sore throat, headache, fatigue (mild to severe), sneezing, night sweats, and are indistinguishable from common cold [17]. Fever, cough, and loss of smell/taste are less common as compared to previous variants. Symptoms usually last for a couple of days followed by quick recovery.

While this is early information, studies from South Africa, England, Scotland, Denmark and Canada have reported significantly lower risk of hospitalization, serious disease, and death in patients with omicron/SGTF infections as compared to delta/non-SGTF infections (Table 1) [18-22].

Hence, evidence so far seems to indicate that omicron is milder in severity than the previous SARS-CoV-2 variants. This is also supported by the fact that despite the surge of infections in South Africa and the UK, the hospitalizations, intensive care admissions, and deaths remained low. In a recently published study from Netcare-a private health care group from South Africa, the characteristics of patients admitted during the four waves were compared [23]. These waves were due to the ancestral virus, beta, delta, and omicron, respectively. The proportion of patients requiring oxygen therapy significantly decreased ( $17.6 \%$ in wave 4 vs. $74 \%$ in wave $3, p<0.001$ ) as did the percentage receiving mechanical ventilation. Admission to intensive care was $18.5 \%$ in wave 4 vs. $29.9 \%$ in wave 3 $(p<0.001)$. The median length of stay (between 7 and $8 \mathrm{~d}$ in previous waves) decreased to $3 \mathrm{~d}$ in wave 4 . The death rate was between $19.7 \%$ in wave 1 and $29.1 \%$ in wave 3 , and decreased to $2.7 \%$ in wave 4 .

However, these are early impressions and the picture may change with time. Information about behavior of omicron in the severely immunocompromised is not available. Also despite the low severity, if the numbers are very high then the testing capacity and health care system will be overwhelmed. The possible impact of omicron on the burden of long COVID should also be kept in mind. Finally, there is the collateral damage from a massive surge of cases to the global economy, attention to other illnesses, mental health of society, and health care workers.

\section{Neutralization by Monoclonal Antibodies}

Monoclonal antibody cocktails including casirivimabimdevimab, bamlanivimab-etesevimab have emerged as 
Table 1 Hospitalization risk with probable/confirmed omicron cases

\begin{tabular}{|c|c|c|}
\hline Place (Reference) & Population & Hospitalization risk \\
\hline South Africa [18] & $\begin{array}{l}38,282 \text { patients tested by the TaqPath PCR between } \\
\text { 1st October to 6th December. } \\
29,721 \text { were SGTF and the rest non-SGTF. }\end{array}$ & $\begin{array}{l}\text { SGTF patients had an adjusted odds ratio of } 0.2(95 \% \text { CI } \\
0.1-0.3) \text { for hospitalization as compared to non-SGTF } \\
\text { patients. } \\
\text { When compared with delta infections in the previous wave, } \\
\text { the odds of severe disease were lower with SGTF patients- } \\
\text { aOR } 0.3(95 \% \text { CI } 0.2-0.5) \text {. }\end{array}$ \\
\hline England [19] & $\begin{array}{l}\text { SGTF and non-SGTF cases between 1st and 14th December, } \\
2021 .\end{array}$ & $\begin{array}{l}\text { The risk of hospitalization with SGTF infections was } 25 \% \\
\text { lower as compared to non-SGTF infections, while it was } 45 \% \\
\text { lower if hospitalization for } 1 \text { or more day was considered. }\end{array}$ \\
\hline Scotland [20] & $\begin{array}{l}23,840 \text { SGTF cases between } 23 \text { rd November and } \\
19 \text { th December. } \\
50 \% \text { cases were in the age group of } 20-39 \text {. }\end{array}$ & $\begin{array}{l}\text { The hospitalization rate was } 15 / 23,840(0.06 \%) \text { giving an } \\
\text { expected/observed ratio of } 0.3 \text {. }\end{array}$ \\
\hline Denmark [21] & $\begin{array}{l}785 \text { cases of omicron till 9th December, age range } 2-95 y, \\
\text { median age } 32 \text { y. }\end{array}$ & $\begin{array}{l}\text { Only } 9 \text { cases were hospitalized, } 1 \text { needed intensive care, and } \\
\text { none died. }\end{array}$ \\
\hline Canada [22] & $\begin{array}{l}6,312 \text { cases of proven or probable omicron and } 8,875 \\
\text { matched delta cases. }\end{array}$ & $\begin{array}{l}\text { The risk of hospitalization was } 0.33 \% \text { and death was } 0 \% \text { with } \\
\text { omicron as compared to } 2.24 \% \text { and } 0.3 \% \text {, respectively with } \\
\text { delta. }\end{array}$ \\
\hline
\end{tabular}

important therapeutics for COVID-19 with demonstrable efficacy in preventing progression to severe disease/hospitalization, treatment of severe disease, and for postexposure prophylaxis [24]. However, in vitro data suggest that the omicron variant completely escapes neutralization by these antibodies [25]. Broadly, neutralizing sarbecovirus monoclonal antibodies including sotrovimab, which attach to sites beyond the receptor binding domain remain effective in vitro against omicron [25]. Sotrovimab is currently not available in India. This escape of omicron from mAbs is relevant for routine clinical practice. In a patient with COVID-19 disease, identification of the variant is important in settings where both delta and omicron are circulating; mAbs would help against delta and other variants but not omicron.

\section{Reinfection Ability}

One of the salient features of omicron is its ability to cause reinfection. Studies before the emergence of omicron showed $0.1 \%-1 \%$ risk of reinfection and prior infection with SARSCoV-2 to give more than $80 \%$ protection against reinfection [26]. Linear data analysis from South Africa showed that while reinfection rates did not go up during the beta and delta waves, they went up significantly during the omicron wave; the hazard ratio for reinfection vs. primary infection between 1 st and 27 th November was 2.39 as compared to wave 1 [18]. One may argue that the reinfection rate was higher with omicron as there was waning of natural immunity acquired during previous infections when omicron struck. However, data from the UK where both delta and omicron are circulating at the same time negates this hypothesis. Here, 6 times higher risk of reinfection with omicron as compared to delta, has been reported. While a previous infection gave $80 \%$ protection against delta, it only gave $19 \%$ protection against omicron [16]. Data from Scotland also show that the proportion of SGTF cases that were possible reinfections were 10 times the proportion of non-SGTF cases that were reinfections [20].

On a positive note, while reinfections are common with omicron, they were associated with a $61 \%$ lower risk of hospitalization as compared to primary infection with omicron in the UK [16]. This indicates that while previous infection may not be strong enough to prevent infection, it mitigates the severity of the disease, a phenomenon similar to vaccineinduced immunity.

\section{Ability to Breakthrough Vaccine-Induced Immunity}

All the currently available vaccines have demonstrated lower neutralizing antibody titers, efficacy, and effectiveness against the variants as compared to the original strain. For example, neutralizing antibody titers induced by the Pfizer (BNT162b2) vaccine were 4-sixfold lower for delta as compared to the original or the alpha variant [27]. Similarly, the effectiveness of two doses of the AstraZeneca (AZD1222) vaccine was $74 \%$ against the alpha variant but dropped to $67 \%$ against the delta variant; the effectiveness of 2 doses of the BNT162b2 vaccine against delta was $88 \%$ as against $94 \%$ for the alpha variant [28]. However, protection against severe disease and hospitalization against delta was more than $90 \%$ with two doses of both the vaccines [29].

Omicron demonstrates a greater breakthrough against vaccine-induced immunity as compared to delta. In vitro studies show that the neutralizing antibody titers induced by BNT162b2 against omicron were 44-fold lower and that by 
AZD1222 were 36-fold lower [25, 30]. Similarly neutralizing antibody titers against omicron were significantly lower with all other vaccines including mRNA-1273 (Moderna), Sputnik, Sinopharm, Ad26.COV2.S (Janssen), etc. Effectiveness data from the UK are also emerging. While the vaccine efficacy against delta with 2 doses of AZD1222 (second dose given more than $2 \mathrm{wk}$ earlier) was $44 \%$, it was- $-5 \%$ with omicron. Corresponding figures for the BNT162b2 were $70 \%$ and $19 \%$, respectively [19].

On the other hand, data from the UK showed that while 2 doses of BNT162b2 or AZD1222 vaccine did not offer protection against symptomatic infection, they did reduce hospitalization due to omicron. Hazard ratios (HR) for hospitalization due to omicron following 2 doses of BNT162b2 and AZD1222 were 0.22 and 0.31 as compared to hospitalization risk with delta in unvaccinated individuals [19]. HR for hospitalization risk for omicron in unvaccinated individuals was 0.59 relative to delta [19]. A study from South Africa showed that 2 doses of BNT162b2 was $70 \%$ protective against hospitalization during the omicron surge as compared to $93 \%$ before the omicron surge [31]. The protection against severe disease/hospitalization with 2 doses of vaccines suggests that cell-mediated immunity is at least partially retained against omicron.

\section{Role of Booster Vaccination Against Omicron}

This is the topic of greatest debate in the current times. Even before omicron emerged, several countries had started to offer boosters to their citizens in response to waning antibody titers 3-6 mo following vaccination [32]. This was despite an appeal by the WHO to ensure vaccination of all adults, especially in low-income countries, with two doses before offering booster doses to its citizens [33]. Most high-income and upper middle-income countries are giving booster doses to its citizens and boosters comprise $25 \%$ of the vaccines administered daily, currently [34].

The argument in favor of boosters against omicron first emerged from in vitro data where the serum of individuals given the booster dose showed an increase in neutralizing antibody titers against omicron [35]. Administration of booster doses of the same vaccine to BNT162b2/mRNA1273 (Moderna) vaccinees caused the neutralizing antibody titers, which were initially 122 -fold/44-fold lower against omicron, to increase to only 4-/sixfold lower after booster. The neutralizing antibody titers were boosted against the Ad26.COV2.S vaccine as well. Simultaneously, information about the effectiveness of boosters emerged from the UK, where almost $50 \%$ of the population has received booster doses [34]. Boosters were given with BNT162b2/mRNA-1273 in both AZD1222/BNT162b2 primed individuals [19]. The vaccine effectiveness against symptomatic infection with omicron increased with the
mRNA booster from-5\% to $73 \%$ in AZD1222 primed individuals, and from 19 to $77 \%$ in those who were primed with BNT162b2 [19]. There was improved protection against hospitalization with omicron in individuals primed with AZD1222 and boosted with mRNA vaccines but not in those who were primed with mRNA vaccines and received mRNA vaccine booster [19].

In view of the increase in cases and fear of an omicron surge, India too announced initiation of booster doses with effect 10th January 2022 to health care workers, frontline workers and those aged above $60 \mathrm{y}$ with the same vaccine as given earlier [36]. However, studies show that heterologous boosters work better than the homologous (same) vaccine [37]. In the COVBOOST study which was a randomized, controlled trial in the UK, humoral and cell- mediated responses against the wild virus and delta were compared with a control group when AZD1222 or BNT162b2 primed individuals were given 7 different booster vaccines [Pfizer, $1 / 2$ dose Pfizer, Moderna, Novavax, Janssen, Valneva (an inactivated vaccine), $1 / 2$ dose Valneva, and Curevac (mRNA vaccine whose development is now stopped)] [38]. The immunogenicity (both humoral/cell-mediated) in Astra Zeneca vaccine primed individuals was best with Moderna $>$ Pfizer $>1 / 2$ dose Pfizer $>$ Novavax $>$ Janssen $>1 / 2$ dose Novavax $>$ AZV in decreasing order. In the Pfizer primed individuals, the best responses were with: Moderna $>$ Pfizer $>1 / 2$ Pfizer $>$ Janssen $>$ AZV $>$ Novavax $>1 / 2$ Novavax in decreasing order. The reactogenicity was most in the Pfizer primed individuals and when Moderna was used as a booster. A booster study in Thailand showed excellent humoral and cell-mediated immune responses when recipients of two doses of Coronavac (an inactivated vaccine) were boosted with a single dose of AZD1222 [39]. Hence, the choice of booster dose in India needs reconsideration.

But apart from the choice of a booster, a bigger question is whether boosters are really needed and will they be effective? And do they justify the cost? The countries with the most booster vaccination coverage (USA, UK, and Europe) are at present seeing a relentless onslaught of omicron, whereas in South Africa where only $30 \%$ of the population has received 2 doses of the vaccine, the cases have started declining spontaneously.

\section{Current Status of the COVID-19 Pandemic in Children}

\section{Global}

The direct effects of SARS-CoV-2 on children have been largely mild and that has remained unchanged even with the variants. Age-disaggregated cases reported to WHO from 30th December 2019 to 25th October 2021 show that 
children under five years of age represent $2 \%$ of reported global cases and $0.1 \%$ of reported global deaths [40]. Older children and younger adolescents [5 to $14 \mathrm{yrs}$ ] account for $7 \%$ of reported global cases and $0.1 \%$ of reported global deaths. Deaths for all ages less than 25 y represented less than $0.5 \%$ of reported global deaths. The fears of future waves preferentially affecting children have, so far, been unfounded. The indirect effects of the pandemic on children have far exceeded the direct effects [41]. Owing to the relatively lower risk of severe disease in children and adolescents, the WHO requested nations to donate vaccines to the COVAX initiative to ensure complete vaccination of all adults before immunization of healthy children [42]. However, COVID-19 vaccination for children/adolescents has been implemented in many countries including US, Canada, Europe including the UK (with BNT162b2) and Middle East (with Sinopharm) [34, 43]. Initially for children aged 12 y and above, it has recently been expanded to include children $5 \mathrm{y}$ and older [43].

With the current surge of omicron in the US, there has been a recent increase in hospitalizations in children infected with COVID-19 both in absolute numbers and as well as a percentage of total COVID hospitalizations [44]. It is, however, not clear whether these hospitalizations are due to severe disease or is it children admitted for other reasons and incidentally detected to be COVID19 infected. Besides the direct effects, the indirect consequences of the omicron surge due to school closures and economic fallout may further worsen the physical, mental, and social health crises in children globally [45]. It is difficult to comment on the impact of the omicron surge on cases of multisystem inflammatory syndrome in children (MIS-C), but no such reports from South Africa have come in yet. However, the pattern over the next few weeks needs to be studied given the lag between the peak of COVID-19 and occurrence of MIS-C.

\section{India}

Children have accounted for $12 \%$ of all cases of COVID19 in India, while they constitute $40 \%$ of the population [46]. More than two-thirds of Indian children were seropositive for COVID-19 in June 2021 suggesting that children were infected to the same extent as adults but were either asymptomatic or not tested [47]. Since the omicron surge in India has just begun, its impact on children is not known yet. Again, the concern here is more of the collateral effects, especially learning and mental health issues of children. India has probably had the longest period of school closures in the world [48]. As the country was readying to open schools and regularize exams, omicron struck. School reopening may again go on the back burner as the system gears itself to meet the other challenges of omicron. The Drug Controller General of India has approved two vaccines for children; ZyCoV-D (a DNA-based vaccine) and Covaxin (an inactivated vaccine) for children aged 12-18 y. The phase I/II safety and immunogenicity study of Covaxin in 526 children aged between 2 and 18 y showed seroconversion in 95\%-98\% of the children, 4 wk after the second dose, superior antibody titers as compared to adults, and acceptable safety and reactogenicity [49]. The Indian government recently announced vaccination of all children aged 15-18 y, beginning January 3, 2022 with Covaxin [36]. While vaccination of children against COVID-19 is welcome, equally or more important is the redressal of other indirect effects of COVID-19 in children [50].

\section{Conclusions}

Omicron has taken the world by storm. It spreads faster and is able to evade natural and vaccine-induced immunity better than its predecessor variants. Despite the milder severity, the public health impact can be considerable due to the sheer number of cases and indirect effects on the global economy. While many privileged nations have resorted to booster vaccines as a strategy to control the spread of this variant, the utility of this strategy is debatable. Whether omicron is going to script another deadly chapter in the history of this pandemic or will it augur an end to the pandemic by replacing the more virulent strains, time alone will tell.

\section{Declarations}

Conflict of Interest None.

\section{References}

1. World Health Organization. WHO Coronavirus (COVID-19) dashboard. Available at: https://covid19.who.int/. Accessed on $29 \mathrm{Dec}$ 2021.

2. Karim SSA, Karim QA. Omicron SARS-CoV-2 variant: a new chapter in the COVID-19 pandemic. Lancet. 2021;398:2126-8.

3. Callaway E. Heavily mutated Omicron variant puts scientists on alert. Nature. 2021;600:21.

4. Torjesen I. Covid-19: Omicron may be more transmissible than other variants and partly resistant to existing vaccines, scientists fear. BMJ. 2021;375:n2943.

5. World Health Organization. Update on omicron. 2021. Available at: www.who.int/news/item/28-11-2021-update-on-omicron. Accessed on 29 Dec 2021.

6. Petersen E, Ntoumi F, Hui DS, et al. Emergence of new SARSCoV-2 variant of concern omicron (B.1.1.529) - highlights Africa's research capabilities, but exposes major knowledge gaps, inequities of vaccine distribution, inadequacies in global 
COVID-19 response and control efforts. Int J Infect Dis. 2021;114:268-72.

7. WorldoMeter. South Africa. 2022. Available at: https://www. worldometers.info/coronavirus/country/south-africa/. Accessed on 29 Dec 2021.

8. Scott L, Hsiao NY, Moyo S, et al. Track omicron's spread with molecular data. Science. 2021;374:1454-5.

9. WorldoMeter. United Kingdom. 2022. Available at: https://www. worldometers.info/coronavirus/country/uk/. Accessed on $29 \mathrm{Dec}$ 2021.

10. BBC. COVID: US reports record infections as Europe's Omicron cases also soar. Available at: https://www.bbc.com/news/world59814661. Accessed on 29 Dec 2021.

11. Johns Hopkins Coronavirus Resource Center. Covid-19 United States Cases by country. Available at: https://coronavirus.jhu.edu/ us-map. Accessed on 29 Dec 2021.

12. The Times of India. Omicron variant live updates: Mumbai, Delhi, Gujarat report big spike in COVID cases. Available at: https:// timesofindia.indiatimes.com/india/covid-vaccination-omicronvariant-india-live-updates-28-december-2021/liveblog/88532102. cms. Accessed on 29 Dec 2021.

13. Majumdar S, Sarkar R. Mutational and phylogenetic analyses of the two lineages of the Omicron variant. J Med Virol. 2021. https://doi.org/10.1002/jmv.27558.

14. Vogel G, Kupferschmidt K. Early lab studies shed light on Omicron's behavior. Science. 2021;374:1543-4.

15. Grabowski F, Kochańczyk M, Lipniacki T. Omicron strain spreads with the doubling time of 3.2-3.6 days in South Africa province of Gauteng that achieved herd immunity to Delta variant. medRxiv. 2021. https://doi.org/10.1101/2021.12.08.21267494

16. Ferguson N, Ghani A, Cori A, et al. Growth, population distribution and immune escape of the Omicron in England. Imperial College London. 2021. https://doi.org/10.25561/93038.

17. Jansen $\mathrm{L}$, Tegomoh $\mathrm{B}$, Lange $\mathrm{K}$, et al. Investigation of a SARS-CoV-2 B.1.1.529 (Omicron) variant cluster - Nebraska, november-december 2021. MMWR Morb Mortal Wkly Rep. 2021;70:1782-4.

18. Wolter N, Jassat W, Walaza S, et al. Early assessment of the clinical severity of the SARS-CoV-2 Omicron variant in South Africa. medRxiv. 2021. https://doi.org/10.1101/2021.12.21.21268116.

19. Ferguson N, Ghani A, Hinsley W, Volz E. Hospitalisation risk for Omicron cases in England. Imperial College London. 2021. Available at: https://www.imperial.ac.uk/media/imperial-college/medicine/ mrc-gida/2021-12-22-COVID19-Report-50.pdf. Accessed on 30 Dec 2021.

20. Sheikh A, Kerr S, Woolhouse M, et al. Severity of Omicron variant of concern and vaccine effectiveness against symptomatic disease: national cohort with nested test negative design study in Scotland. 2021. Available at: https://politi.co/3EqDs2y. Accessed on 30 Dec 2021.

21. Espenhain L, Funk T, Overvad M, et al. Epidemiological characterisation of the first 785 SARS-CoV-2 Omicron variant cases in Denmark, December 2021. Euro Surveill. 2021;26. https://doi. org/10.2807/1560-7917.ES.2021.26.50.2101146.

22. Ontario Agency for Health Protection and Promotion (Public Health Ontario). Early estimates of Omicron severity in Ontario based on a matched cohort study, November 22 to December 17, 2021. Toronto, ON: Queen's Printer for Ontario; 2021. Available at: https://www.publichealthontario.ca/-/media/documents/ncov/ epi/covid-19-epi-enhanced-estimates-omicron-severity-study.pdf? sc_lang=en. Accessed on 30 Dec 2021.

23. Maslo C, Friedland R, Toubkin M, Laubscher A, Akaloo T, Kama B. Characteristics and outcomes of hospitalized patients in South Africa during the COVID-19 omicron wave compared with previous waves. JAMA. 2021. https://doi.org/10.1001/jama.2021. 24868.
24. Hurt AC, Wheatley AK. Neutralizing antibody therapeutics for COVID-19. Viruses. 2021;13:628.

25. Cameroni E, Saliba C, Bowen JE, et al. Broadly neutralizing antibodies overcome SARS-CoV-2 Omicron antigenic shift. bioRxiv. 2021. https://doi.org/10.1101/2021.12.12.472269.

26. O Murchu E, Byrne P, Carty PG, et al. Quantifying the risk of SARS-CoV-2 reinfection over time. Rev Med Virol. 2021. https:// doi.org/10.1002/rmv.2260.

27. Noori M, Nejadghaderi SA, Arshi S, et al. Potency of BNT162b2 and mRNA-1273 vaccine-induced neutralizing antibodies against severe acute respiratory syndrome-CoV-2 variants of concern: A systematic review of in vitro studies. Rev Med Virol. 2021. https:// doi.org/10.1002/rmv.2277.

28. Lopez Bernal J, Andrews N, Gower C, et al. Effectiveness of COVID-19 Vaccines against the B.1.617.2 (Delta) Variant. N Engl J Med. 2021;385:585-94.

29. Public Health England. Vaccines highly effective against hospitalisation from Delta variant. Available at: https://www.gov.uk/government/ news/vaccines-highly-effective-against-hospitalisation-from-deltavariant. 2021. Accessed on 30 Dec 2021.

30. Dejnirattisai W, Shaw RH, Supasa P, et al. Com-COV2 study group. Reduced neutralisation of SARS-CoV-2 omicron B.1.1.529 variant by post-immunisation serum. Lancet. 2021. https://doi.org/ 10.1016/S0140-6736(21)02844-0.

31. Collie S, Champion J, Moultrie H, Bekker LG, Gray G. Effectiveness of BNT162b2 vaccine against Omicron variant in South Africa. N Engl J Med. 2021. https://doi.org/10.1056/NEJMc2119270.

32. Goldberg Y, Mandel M, Bar-On YM, et al. Waning immunity after the BNT162b2 vaccine in Israel. N Engl J Med. 2021;385:e85.

33. Chagla Z, Pai M. COVID-19 boosters in rich nations will delay vaccines for all. Nat Med. 2021;27:1659-60.

34. Our World in Data. Coronavirus (COVID-19) vaccinations. Available at: https://ourworldindata.org/covid-vaccinations. Accessed on 30 Dec 2021.

35. Garcia-Beltran WF, St. Denis KJ, Hoelzemer A, et al. mRNAbased COVID-19 vaccine boosters induce neutralizing immunity against SARS-CoV-2 Omicron variant. medRxiv. https://doi.org/ 10.1101/2021.12.14.21267755.

36. Ministry of Health and Family Welfare, Government of India. Guidelines for COVID-19 vaccination of children between 15-18 years and precaution dose to HCWs, FLWs \& 60+ population with comorbidities. Available at: https://www.mohfw.gov.in/pdf/ GuidelinesforCOVID19VaccinationofChildrenbetween 15to18y earsandPrecautionDosetoHCWsFLWs\&60populationwithcomo rbidities.pdf. Accessed on 30 Dec 2021.

37. Chiu NC, Chi H, Tu YK, et al. To mix or not to mix? A rapid systematic review of heterologous prime-boost covid-19 vaccination. Expert Rev Vaccines. 2021;20:1211-20.

38. Munro APS, Janani L, Cornelius V, et al. Safety and immunogenicity of seven COVID-19 vaccines as a third dose (booster) following two doses of ChAdOx1 nCov-19 or BNT162b2 in the UK (COV-BOOST): a blinded, multicentre, randomised, controlled, phase 2 trial. Lancet. 2021;398:2258-76.

39. Yorsaeng R, Suntronwong N, Phowatthanasathian H, et al. Immunogenicity of a third dose viral-vectored COVID-19 vaccine after receiving two-dose inactivated vaccines in healthy adults. Vaccine. 2021. https://doi.org/10.1016/j.vaccine.2021.11.083.

40. World Health Organization. COVID-19 disease in children and adolescents: Scientific brief. 2021. Available at: https://www.who. int/publications/i/item/WHO-2019-nCoV-Sci_Brief-Children_ and_adolescents-2021.1. Accessed on 30 Dec 2021.

41. Howard-Jones AR, Bowen AC, Danchin M, et al. COVID-19 in children: I. Epidemiology, prevention and indirect impacts. J Paediatr Child Health. 2021. https://doi.org/10.1111/jpc.15791.

42. World Health Organization. Interim statement on COVID-19 vaccination for children and adolescents. Available at: https://www. 
who.int/news/item/24-11-2021-interim-statement-on-covid-19vaccination-for-children-and-adolescents. Accessed on $30 \mathrm{Dec}$ 2021.

43. Centers for Disease Control and Prevention. COVID-19 Vaccines for Children and Teens. 2021. Available at: https://www.cdc.gov/ coronavirus/2019-ncov/vaccines/recommendations/childrenteens.html. Accessed on 30 Dec 2021.

44. Aljazeera. Omicron is sending thousands of children to US hospitals. 2021. Available at: https://www.aljazeera.com/news/2021/ 12/30/omicron-is-sending-thousands-of-children-to-us-hospitals. Accessed on 31 Dec 2021.

45. Guardian. US alarm at rise in child COVID infections sees school closures back on agenda. 2021. Available at: https:// www.theguardian.com/world/2021/dec/27/us-child-covidomicron-infections-school-closures. Accessed on 30 Dec 2021.

46. Statista. Number of the coronavirus (COVID-19) cases in India as of October 18, 2021, by age group. Available at: https://www.statista. com/statistics/1110522/india-number-of-coronavirus-cases-by-agegroup/. Accessed on 30 Dec 2021.

47. Misra P, Kant S, Guleria R, et al. WHO Unity Serological prevalence study team of AIIMS. Serological prevalence of
SARS-CoV-2 antibody among children and young age (between age 2-17 years) group in India: An interim result from a large multi-centric population-based seroepidemiological study. medRxiv. https://doi.org/10.1101/2021.06.15.21258880

48. UNICEF. COVID-19: Schools for more than 168 million children globally have been completely closed for almost a full year, says UNICEF. Available at: https://www.unicef.org/india/pressreleases/covid-19-schools-more-168-million-children-globallyhave-been-completely-closed. Accessed on 30 Dec 2021.

49. Vadrevu KM, Reddy S, Jogdand H, et al. Immunogenicity and safety of an inactivated SARS-CoV-2 vaccine (BBV152) in children from 2 to 18 years of age: an open-label, age-de-escalation phase 2/3 study. medRxiv. https://doi.org/10.1101/2021.12.28. 21268468.

50. Chanchlani N, Buchanan F, Gill PJ. Addressing the indirect effects of COVID-19 on the health of children and young people. CMAJ. 2020;192:E921-7.

Publisher's Note Springer Nature remains neutral with regard to jurisdictional claims in published maps and institutional affiliations. 